\title{
COMPARISONS OF PARALLEL SYSTEMS ACCORDING TO THE CONVEX TRANSFORM ORDER
}

\author{
SUBHASH KOCHAR $* * *$ AND \\ MAOCHAO XU, ${ }^{* * *}$ Portland State University
}

\begin{abstract}
A parallel system with heterogeneous exponential component lifetimes is shown to be more skewed (according to the convex transform order) than the system with independent and identically distributed exponential components. As a consequence, equivalent conditions for comparing the variabilities of the largest order statistics from heterogeneous and homogeneous exponential samples in the sense of the dispersive order and the rightspread order are established. A sufficient condition is also given for the proportional hazard rate model.
\end{abstract}

Keywords: Convex transform order; exponential distribution; increasing failure rate; parallel system; proportional hazard rate; right-spread order; skewness

2000 Mathematics Subject Classification: Primary 60E15; 62N05; 62G30; 62D05

\section{Introduction}

In statistics, skewness describes the departure of a density from symmetry, where one tail of the density is 'stretched out' more than the other. Several well-known measures of skewness are available in the statistics literature, such as Pearson's coefficient of skewness and Edgeworth's coefficient. Interested readers may refer to Arnold and Groeneveld (1995) and Marshall and Olkin (2007, p. 70) for more discussion and other measures of skewness. In the case of unimodal distributions, if a density is to the left of 'center' and the right tail is relatively long, then the density is said to be skewed to the right. This kind of skewness is used when the distributions have nonnegative support. It is of interest to compare the skewness of two distributions. Van Zwet (1970) proposed a skewness order, called the convex transform order, which captures the property of one distribution being more skewed than the other. This order is also an important concept in reliability theory, since it reflects one distribution being more IFR (increasing failure rate) than the other (more discussion will be given in Section 2).

Series and parallel systems are the building blocks of more complex coherent systems. The life of a parallel system is the same as that of the largest order statistic and the life of a series system is the same as that of the smallest order statistic. Note that when the components are independent, the hazard rate of a series system is the sum of the hazard rates of the components. In particular, if the components are independent and identically distributed, the ageing properties of a series system will be the same as those of the components. But this is not the case with parallel systems. In fact, it is well known that the lifetime of a parallel system with independent IFR components may not be IFR if they are not identically distributed.

Received 10 October 2008; revision received 10 February 2009.

* Postal address: Department of Mathematics and Statistics, Portland State University, Portland, Oregon 97201, USA.

** Email address: kochar@pdx.edu

*** Email address: maochao@pdx.edu 
Consider two parallel systems with independent exponential components, one with identical components and the other with nonidentical components. Many authors have studied the magnitude (stochastic) and variability orderings of such systems when the parameters of the exponential distributions satisfy certain restrictions. For example, Dykstra et al. (1997) showed that if $X_{1}, \ldots, X_{n}$ are independent exponential random variables with $X_{i}$ having hazard rate $\lambda_{i}, i=1, \ldots, n$, and if $Y_{1}, \ldots, Y_{n}$ is a random sample of size $n$ from an exponential distribution with common hazard rate $\lambda=\sum_{i=1}^{n} \lambda_{i} / n$, the arithmetic mean of the $\lambda_{i} \mathrm{~s}$, then

$$
Y_{n: n} \leq_{\mathrm{hr}} X_{n: n} \quad \text { and } \quad Y_{n: n} \leq_{\operatorname{disp}} X_{n: n}
$$

where $X_{n: n}$ and $Y_{n: n}$ are the largest order statistics of the $X_{i}$ s and $Y_{i}$ s, respectively. Here ' $\leq \mathrm{hr}$ ' and ' $\leq_{\text {disp' }}$ denote the hazard rate and dispersive orders, respectively. Khaledi and Kochar (2000) pointed out that the above results also hold under the weaker condition when the common parameter of the $Y_{i} \mathrm{~s}$ is $\lambda=\left(\prod_{i=1}^{n} \lambda_{i}\right)^{1 / n}$, the geometric mean of the $\lambda_{i} \mathrm{~s}$. Recently, Kochar and $\mathrm{Xu}(2007 \mathrm{~b})$ proved that the relationship in (1.1) could be strengthened from the hazard rate order to the likelihood ratio order. We refer the reader to Kochar and Xu (2007a) for a review of this topic.

In this paper we will compare such systems from a different perspective of statistics through the skewness order. More precisely, two such systems will be compared according to the convex transform order, which compares the shapes of their probability distributions. As we discuss below, it can also be interpreted as comparing the relative ageing of two distributions. To the best of the authors' knowledge, this problem has not been studied in the literature and the purpose of this paper is to fill this gap. Intuitively, the density function of the largest order statistic from a heterogeneous sample will be more skewed than one from a homogeneous sample. This will be confirmed in this paper according to the convex transform order for exponential samples. While the previous work mentioned above gives us bounds on measures of variability, such as the variance of the largest order statistic in terms of the independent and identically distributed case, the new result will give us bounds on measures of skewness, such as the coefficient of variation. It will be shown that the coefficient of variation of the largest order statistic of $n$ independent and nonidentically distributed exponential random variables satisfies the inequality

$$
\operatorname{cv}\left(X_{n: n}\right) \geq\left(\sqrt{\sum_{i=1}^{n} \frac{1}{i^{2}}}\right) /\left(\sum_{i=1}^{n} \frac{1}{i}\right) .
$$

Using our main result, in Proposition 4.1 we give a characterization of the dispersion relation in (1.1). We prove that the dispersive order there is equivalent to the usual stochastic order, which gives us a deeper understanding of the result in Khaledi and Kochar (2000). An equivalent characterization for comparing systems in terms of the right-spread order, which can be considered as the second variability order, is also derived as a consequence.

As pointed out in Khaledi and Kochar (2002) and Kochar and Xu (2007b), (1.1) can be extended to the proportional hazard rate (PHR) model, where $X_{1}, \ldots, X_{n}$ are mutually independent lifetimes with $X_{i}$ having survival function $\bar{F}^{\lambda_{i}}, i=1, \ldots, n$, and $Y_{1}, \ldots, Y_{n}$ is a random sample from a distribution with common survival distribution $\bar{F}^{\lambda}$. A sufficient condition for the right-spread order is derived under this scheme, which complements the dispersive ordering result in the literature.

The rest of paper is organized as follows. In Section 2 we introduce some notation and stochastic orders. The convex transform order between the homogeneous and heterogeneous 
distributions of the largest order statistics is studied in Section 3. In Section 4 we give equivalent conditions for the dispersive order and right-spread order of the largest order statistics. A sufficient condition for the right-spread order in the PHR models is also established there.

\section{Preliminaries}

Assume that the random variables $X$ and $Y$ have distribution functions $F$ and $G$, survival functions $\bar{F}=1-F$ and $\bar{G}=1-G$, density functions $f$ and $g$, and failure rate functions $r_{X}=f / \bar{F}$ and $r_{Y}=g / \bar{G}$, respectively.

Definition 2.1. The random variable $X$ is said to be smaller than $Y$ in the convex transform order, denoted by $X \leq_{\mathrm{c}} Y$, if and only if $G^{-1} F(x)$ is convex in $x$ on the support of $X$, where $G^{-1}$ denotes the right-continuous inverse.

If $X \leq_{\mathrm{c}} Y$ then $Y$ is more skewed than $X$, as explained in van Zwet (1970) and Marshall and Olkin (2007, p. 70). The convex transform order is also called the more IFR order in reliability theory, since when $f$ and $g$ exist, the convexity of $G^{-1} F(x)$ means that

$$
\frac{f\left(F^{-1}(u)\right)}{g\left(G^{-1}(u)\right)}=\frac{r_{X}\left(F^{-1}(u)\right)}{r_{Y}\left(G^{-1}(u)\right)}
$$

is increasing in $u \in[0,1]$. Thus, $X \leq_{\mathrm{c}} Y$ can be interpreted to mean that $X$ ages faster than $Y$ in some sense. This partial order is scale invariant. Note that neither $F$ nor $G$ need to be IFR for this definition to hold. It can be seen that $X$ is an IFR distribution if and only if it is convex ordered with respect to the exponential distribution, which has a constant failure rate. It is known that if $X_{\alpha_{i}}$ has gamma distribution with shape parameter $\alpha_{i}, i=1,2$, then $X_{\alpha_{1}} \leq_{\mathrm{c}} X_{\alpha_{2}}$ for $\alpha_{2} \leq \alpha_{1}$.

In the literature, several weaker orders have also been proposed to compare the relative ageing properties. These are the star transform order, denoted by ' $\leq_{\star}$ ', the superadditive order, denoted by ' $\leq$ su', the DMRL (decreasing mean residual lifetime) order, denoted by ' $\leq$ DMRL', the NBUE (new better than used in expectation) order, denoted by ' $\leq$ NBUE', and the HNBUE (harmonic NBUE) order. It has also been pointed out in Kochar (1989) that the HNBUE order is equivalent to the Lorenz order (denoted by ' $\leq$ Lorenz'), a well-known order in economics. We have the following chain of implications:

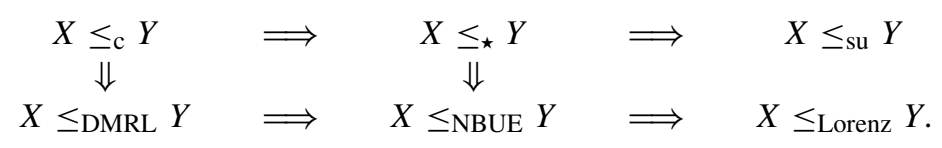

It is also known that (see Marshall and Olkin (2007, p. 69))

$$
X \leq_{\text {Lorenz }} Y \quad \Longrightarrow \quad \mathrm{cv}(X) \leq \mathrm{cv}(Y)
$$

where $\operatorname{cv}(X)$ and $\operatorname{cv}(Y)$ denote the coefficients of variation of $X$ and $Y$, respectively.

A good discussion of the convex, star, and superadditive transform orders can be found in Barlow and Proschan (1981, p. 107) and Marshall and Olkin (2007, p. 70). The DMRL, NBUE, and HNBUE partial orders were introduced in Kochar and Wiens (1987) and studied further in Kochar (1989) and Kochar et al. (2002).

A basic partial order to compare the variabilities in the two distributions is the dispersive ordering, defined below. 
Definition 2.2. The random variable $X$ is said to be less dispersed than $Y$ (denoted by $X \leq_{\text {disp }}$ $Y)$ if

$$
F^{-1}(\beta)-F^{-1}(\alpha) \leq G^{-1}(\beta)-G^{-1}(\alpha) \text { for all } 0<\alpha \leq \beta<1,
$$

where $F^{-1}$ and $G^{-1}$ denote their corresponding right-continuous inverses.

A weaker order called the right-spread order in Fernández-Ponce et al. (1998), or the excess wealth order in Shaked and Shanthikumar (1998), has also been proposed to compare the variabilities of the two distributions.

Definition 2.3. The random variable $X$ is said to be less right spread than $Y$ (denoted by $\left.X \leq{ }_{\mathrm{RS}} Y\right)$ if

$$
\int_{F^{-1}(p)}^{\infty} \bar{F}(x) \mathrm{d} x \leq \int_{G^{-1}(p)}^{\infty} \bar{G}(x) \mathrm{d} x \quad \text { for all } 0 \leq p \leq 1 .
$$

For more discussion on the dispersive order and the right-spread order, we refer the reader to Shaked and Shanthikumar (2007, Sections 3.B and 3.C) and the references therein.

The usual stochastic order is also discussed in the sequel.

Definition 2.4. The random variable $X$ is said to be smaller than $Y$ in the usual stochastic order (denoted by $X \leq$ st $Y$ ) if $\bar{F}(x) \leq \bar{G}(x)$ for all $x$.

\section{Which parallel system ages faster?}

Suppose that we have two parallel systems, one with independent and identically distributed exponential components and the other with heterogeneous exponential components. The following result shows that a parallel system with homogeneous exponential components ages faster than the one with heterogeneous exponential components in the sense of the convex transform order.

Theorem 3.1. Let $X_{1}, \ldots, X_{n}$ be independent exponential random variables with $X_{i}$ having hazard rate $\lambda_{i}, i=1, \ldots, n$. Let $Y_{1}, \ldots, Y_{n}$ be a random sample of size $n$ from an exponential distribution with common hazard rate $\lambda$. Then,

$$
Y_{n: n} \leq_{\mathrm{c}} X_{n: n} .
$$

Proof. Let $G$ and $F$ denote the distribution functions of $Y_{n: n}$ and $X_{n: n}$ with corresponding density functions $g$ and $f$, respectively.

Then, for $x \geq 0$,

$$
G(x)=\mathrm{P}\left(Y_{n: n} \leq x\right)=\left(1-\mathrm{e}^{-\lambda x}\right)^{n}
$$

and

$$
F(x)=\mathrm{P}\left(X_{n: n} \leq x\right)=\prod_{i=1}^{n}\left(1-\mathrm{e}^{-\lambda_{i} x}\right) .
$$

From Proposition 21.A.7 of Marshall and Olkin (2007), it is sufficient to show that $G^{-1} F(x)$ is concave on $(0, \infty)$. Note that, for $x \geq 0$,

$$
G^{-1} F(x)=-\frac{1}{\lambda} \ln \left(1-F^{1 / n}(x)\right)=-\frac{1}{\lambda} \ln \left(1-\prod_{i=1}^{n}\left(1-\mathrm{e}^{-\lambda_{i} x}\right)^{1 / n}\right) .
$$


Hence,

$$
g\left(G^{-1} F(x)\right)=n \lambda\left(1-\prod_{i=1}^{n}\left(1-\mathrm{e}^{-\lambda_{i} x}\right)^{1 / n}\right) \prod_{i=1}^{n}\left(1-\mathrm{e}^{-\lambda_{i} x}\right)^{(n-1) / n} .
$$

Differentiating with respect to $x$, we obtain

$$
\begin{aligned}
\left(G^{-1}\right. & F(x))^{\prime} \\
& =\frac{f(x)}{g\left(G^{-1} F(x)\right)} \\
& =\sum_{i=1}^{n} \frac{\lambda_{i} \mathrm{e}^{-\lambda_{i} x}}{1-\mathrm{e}^{-\lambda_{i} x}} \prod_{i=1}^{n}\left(1-\mathrm{e}^{-\lambda_{i} x}\right) / n \lambda\left[1-\prod_{i=1}^{n}\left(1-\mathrm{e}^{-\lambda_{i} x}\right)^{1 / n}\right] \prod_{i=1}^{n}\left(1-\mathrm{e}^{-\lambda_{i} x}\right)^{(n-1) / n} \\
& =\sum_{i=1}^{n} \frac{\lambda_{i} \mathrm{e}^{-\lambda_{i} x}}{1-\mathrm{e}^{-\lambda_{i} x}} \prod_{i=1}^{n}\left(1-\mathrm{e}^{-\lambda_{i} x}\right)^{1 / n} / n \lambda\left(1-\prod_{i=1}^{n}\left(1-\mathrm{e}^{-\lambda_{i} x}\right)^{1 / n}\right)
\end{aligned}
$$

So, it is enough to prove that

$$
h(x)=\frac{\sum_{i=1}^{n} \lambda_{i} \mathrm{e}^{-\lambda_{i} x} /\left(1-\mathrm{e}^{-\lambda_{i} x}\right)}{\prod_{i=1}^{n}\left(1-\mathrm{e}^{-\lambda_{i} x}\right)^{-1 / n}-1}=\frac{\sum_{i=1}^{n} \lambda_{i} /\left(\mathrm{e}^{\lambda_{i} x}-1\right)}{\prod_{i=1}^{n}\left(1-\mathrm{e}^{-\lambda_{i} x}\right)^{-1 / n}-1}
$$

is decreasing in $x \geq 0$. By taking the derivative, it is equivalent to showing that

$$
\begin{aligned}
& \sum_{i=1}^{n} \frac{\lambda_{i}^{2} \mathrm{e}^{\lambda_{i} x}}{\left(\mathrm{e}^{\lambda_{i} x}-1\right)^{2}}\left(\prod_{i=1}^{n}\left(1-\mathrm{e}^{-\lambda_{i} x}\right)^{-1 / n}-1\right) \\
& \quad \geq \frac{1}{n} \sum_{i=1}^{n} \frac{\lambda_{i}}{\mathrm{e}^{\lambda_{i} x}-1} \sum_{i=1}^{n} \frac{\lambda_{i} \mathrm{e}^{-\lambda_{i} x}}{1-\mathrm{e}^{-\lambda_{i} x}} \prod_{i=1}^{n}\left(1-\mathrm{e}^{-\lambda_{i} x}\right)^{-1 / n},
\end{aligned}
$$

i.e.

$$
\sum_{i=1}^{n} \frac{\lambda_{i}^{2} \mathrm{e}^{-\lambda_{i} x}}{\left(1-\mathrm{e}^{-\lambda_{i} x}\right)^{2}}\left(1-\prod_{i=1}^{n}\left(1-\mathrm{e}^{-\lambda_{i} x}\right)^{1 / n}\right) \geq \frac{1}{n}\left(\sum_{i=1}^{n} \frac{\lambda_{i} \mathrm{e}^{-\lambda_{i} x}}{1-\mathrm{e}^{-\lambda_{i} x}}\right)^{2} .
$$

From the classical Cauchy-Schwarz inequality (see Mitrinović (1970, Theorem 1, p.41)), it follows that, for $x \geq 0$,

$$
\sum_{i=1}^{n} \frac{\lambda_{i}^{2} \mathrm{e}^{-\lambda_{i} x}}{\left(1-\mathrm{e}^{-\lambda_{i} x}\right)^{2}} \sum_{i=1}^{n} \mathrm{e}^{-\lambda_{i} x} \geq\left(\sum_{i=1}^{n} \frac{\lambda_{i} \mathrm{e}^{-\lambda_{i} x}}{1-\mathrm{e}^{-\lambda_{i} x}}\right)^{2}
$$

Hence, it is sufficient to show that, for $x \geq 0$,

$$
\sum_{i=1}^{n} \frac{\lambda_{i}^{2} \mathrm{e}^{-\lambda_{i} x}}{\left(1-\mathrm{e}^{-\lambda_{i} x}\right)^{2}}\left(1-\prod_{i=1}^{n}\left(1-\mathrm{e}^{-\lambda_{i} x}\right)^{1 / n}\right) \geq \frac{1}{n} \sum_{i=1}^{n} \frac{\lambda_{i}^{2} \mathrm{e}^{-\lambda_{i} x}}{\left(1-\mathrm{e}^{-\lambda_{i} x}\right)^{2}} \sum_{i=1}^{n} \mathrm{e}^{-\lambda_{i} x},
$$

i.e.

$$
\frac{1}{n} \sum_{i=1}^{n}\left(1-\mathrm{e}^{-\lambda_{i} x}\right) \geq \prod_{i=1}^{n}\left(1-\mathrm{e}^{-\lambda_{i} x}\right)^{1 / n}
$$

which is guaranteed by the arithmetic-geometric mean inequality. 


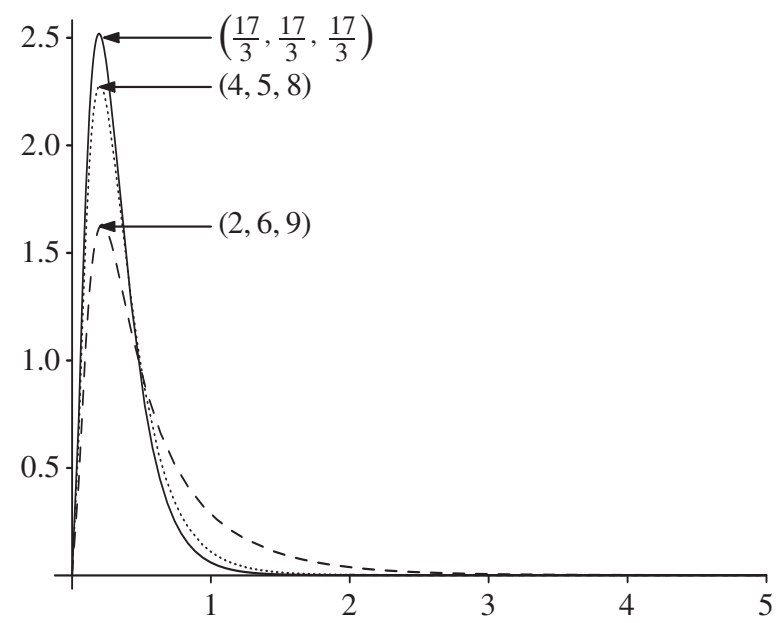

Figure 1: The densities of $X_{n: n}$ with $\left(\lambda_{1}, \lambda_{2}, \lambda_{3}\right)=(2,6,9)$ (dashed line), $X_{n: n}$ with $\left(\lambda_{1}, \lambda_{2}, \lambda_{3}\right)=$ $(4,5,8)$ (dotted line), and $Y_{n: n}$ with $\lambda=\frac{17}{3}$ (solid line).

Remark 3.1. Theorem 3.1 reveals that a parallel system with homogeneous exponential components ages faster than a system with heterogeneous exponential components in the sense of the 'more IFR' property. Note that a parallel system with homogeneous exponential components is IFR (see Barlow and Proschan (1981, p. 108)). However, a parallel system with heterogeneous exponential components may not be IFR.

Remark 3.2. It is interesting to note that, unlike the magnitude and variability orders, no restriction on the parameters is needed for Theorem 3.1 to hold as the convex transform order is scale invariant. Intuitively, owing to the heterogeneity, the largest order statistic from a heterogeneous sample will be more skewed than that from a homogeneous sample. Theorem 3.1 confirms this fact for exponential samples.

In Figure 1 we plot the densities of three parallel systems with independent exponential components and with parameters $(2,6,9),(4,5,8)$, and $\left(\frac{17}{3}, \frac{17}{3}, \frac{17}{3}\right)$. It is easy to verify that

$$
\left(\frac{17}{3}, \frac{17}{3}, \frac{17}{3}\right) \prec(4,5,8) \prec(2,6,9)
$$

in the majorization ordering of Marshall and Olkin (1979). Let $X_{1}, \ldots, X_{n}$ be independent exponential random variables with $X_{i}$ having hazard rate $\lambda_{i}, i=1, \ldots, n$, and let $Y_{1}, \ldots, Y_{n}$ be a random sample of size $n$ from an exponential distribution with $Y_{i}$ having hazard rate $\theta_{i}, i=1, \ldots, n$, such that

$$
\left(\theta_{1}, \ldots, \theta_{n}\right) \prec\left(\lambda_{1}, \ldots, \lambda_{n}\right) .
$$

Looking at Figure 1, one may wonder whether in this case

$$
Y_{n: n} \leq_{\mathrm{c}} X_{n: n}
$$

Though we believe this result to be true from empirical evidence, we are unable to establish it mathematically so far.

The following result, which is of independent interest in economics, is a direct consequence of Theorem 3.1. 
Corollary 3.1. Let $X_{1}, \ldots, X_{n}$ be independent exponential random variables with $X_{i}$ having hazard rate $\lambda_{i}, i=1, \ldots, n$. Let $Y_{1}, \ldots, Y_{n}$ be a random sample of size $n$ from an exponential distribution with common hazard rate $\lambda$. Then,

$$
Y_{n: n} \leq \text { Lorenz } X_{n: n} .
$$

From Barlow and Proschan (1981, p. 60), it follows that

$$
\mathrm{E} Y_{n: n}=\frac{1}{\lambda} \sum_{i=1}^{n} \frac{1}{i}
$$

and

$$
\operatorname{var}\left(Y_{n: n}\right)=\frac{1}{\lambda^{2}} \sum_{i=1}^{n} \frac{1}{i^{2}}
$$

Using (2.1), we obtain the following lower bound on the coefficient of variation of $X_{n: n}$ :

$$
\operatorname{cv}\left(X_{n: n}\right) \geq\left(\sqrt{\sum_{i=1}^{n} \frac{1}{i^{2}}}\right) /\left(\sum_{i=1}^{n} \frac{1}{i}\right) .
$$

\section{Dispersion}

The next result gives equivalent characterizations of the dispersive order and the right-spread order between two parallel systems.

Proposition 4.1. Let $X_{1}, \ldots, X_{n}$ be independent exponential random variables with $X_{i}$ having hazard rate $\lambda_{i}, i=1, \ldots, n$, and let $Y_{1}, \ldots, Y_{n}$ be a random sample of size $n$ from an exponential distribution with common hazard rate $\lambda$. Then

(i) $Y_{n: n} \leq_{\text {disp }} X_{n: n}$ if and only if $Y_{n: n} \leq_{\mathrm{st}} X_{n: n}$;

(ii) $Y_{n: n} \leq{ }_{\mathrm{RS}} X_{n: n}$ if and only if $\mathrm{E} Y_{n: n} \leq \mathrm{E} X_{n: n}$.

Proof. (i) Ahmed et al. (1986) proved in their Theorem 3 that the conditions $Y \leq_{\mathrm{su}} X$ and $Y \leq_{\text {st }} X$ imply that $Y \leq_{\text {disp }} X$. Since $Y_{n: n} \leq_{\mathrm{c}} X_{n: n}$ implies that $Y_{n: n} \leq_{\text {su }} X_{n: n}$, it follows that the condition $Y_{n: n} \leq_{\text {st }} X_{n: n}$ will imply that $Y_{n: n} \leq_{\text {disp }} X_{n: n}$. Conversely, observing that $Y_{n: n} \leq_{\text {disp }} X_{n: n}$ implies that $Y_{n: n} \leq_{\text {st }} X_{n: n}$ in the case of distributions with a common left-hand point of the support, the required result follows immediately from Theorem 3.1.

(ii) Theorem 4.3 of Fernández-Ponce et al. (1998) shows that $Y \leq \mathrm{NBUE} X$ and E $Y \leq \mathrm{E} X$ imply that $Y \leq_{\mathrm{RS}} X$. Note that $Y_{n: n} \leq_{\mathrm{c}} X_{n: n}$ implies that $Y_{n: n} \leq_{\mathrm{NBUE}} X_{n: n}$ and, since $Y_{n: n} \leq_{\mathrm{RS}} X_{n: n}$ implies that $\mathrm{E} Y_{n: n} \leq \mathrm{E} X_{n: n}$, the result follows from Theorem 3.1 again.

Khaledi and Kochar (2000) proved that

$$
\lambda=\left(\prod_{i=1}^{n} \lambda_{i}\right)^{1 / n} \Longrightarrow Y_{n: n} \leq_{\operatorname{disp}} X_{n: n} .
$$

Actually, we may easily check that

$$
\hat{\lambda}=\left(\prod_{i=1}^{n} \lambda_{i}\right)^{1 / n} \leq \lambda \quad \Longleftrightarrow \quad Y_{n: n} \leq \operatorname{disp} X_{n: n} .
$$


Now, one question arises naturally: what is the equivalent condition for the right-spread order between these two parallel systems? This question has not been addressed for a long time since it is not straightforward to check using the definition of the right-spread order. However, using Proposition 4.1, we could easily solve this problem.

Proposition 4.2. Let $X_{1}, \ldots, X_{n}$ be independent exponential random variables with $X_{i}$ having hazard rate $\lambda_{i}, i=1, \ldots, n$. Let $Y_{1}, \ldots, Y_{n}$ be a random sample of size $n$ from an exponential distribution with common hazard rate $\lambda$. Then,

$$
\lambda^{*} \leq \lambda \quad \Longleftrightarrow \quad Y_{n: n} \leq \mathrm{RS} X_{n: n},
$$

where

$$
\lambda^{*}=\sum_{i=1}^{n} \frac{1}{i}\left(\sum_{k=1}^{n}(-1)^{k+1} \sum_{1 \leq i_{1} \leq \cdots \leq i_{k} \leq n} \frac{1}{\sum_{j=1}^{k} \lambda_{i_{j}}}\right)^{-1} .
$$

Proof. Note that

$$
\mathrm{E} Y_{n: n}=\frac{1}{\lambda} \sum_{i=1}^{n} \frac{1}{i}
$$

and, for $x \geq 0$,

$$
\begin{aligned}
\mathrm{E} X_{n: n} & =\int_{0}^{\infty}\left(1-\prod_{i=1}^{n}\left(1-\mathrm{e}^{-\lambda_{i} x}\right)\right) \mathrm{d} x \\
& =\int_{0}^{\infty}\left(\sum_{k=1}^{n}(-1)^{k+1} \sum_{1 \leq i_{1} \leq \cdots \leq i_{k} \leq n} \exp \left[-\sum_{j=1}^{k} \lambda_{i_{j}} x\right]\right) \mathrm{d} x \\
& =\sum_{k=1}^{n}(-1)^{k+1} \sum_{1 \leq i_{1} \leq \cdots \leq i_{k} \leq n} \frac{1}{\sum_{j=1}^{k} \lambda_{i_{j}}} .
\end{aligned}
$$

Hence, the result follows immediately from Proposition 4.1(ii).

From (4.2) and (4.3), it is seen that $\lambda^{*} \leq \hat{\lambda}$ as the dispersive order implies the right-spread order. Although it is complicated to compute $\lambda^{*}$, Proposition 4.2 is very useful since E $X_{n: n}$ could either be easily numerically computed or derived directly from the data. The following example gives a good illustration.

Example 4.1. Let $\lambda_{1}=2, \lambda_{2}=4$, and $\lambda_{3}=5$. Now,

$$
E X_{3: 3}=\int_{0}^{\infty}\left(1-\prod_{i=1}^{n}\left(1-\mathrm{e}^{-\lambda_{i} x}\right)\right) \mathrm{d} x=\frac{8597}{13860}
$$

Hence,

$$
\lambda^{*}=\frac{13860}{8597}\left(1+\frac{1}{2}+\frac{1}{3}\right)=2.95568
$$

However,

$$
\hat{\lambda}=(40)^{1 / 3}=3.41995
$$

So $\lambda^{*}<\hat{\lambda}$. 
Relation (4.1) has been extended to the PHR model in Khaledi and Kochar (2002). Let $X_{1}, \ldots, X_{n}$ be independent random variables with $X_{i}$ having survival function $\bar{F}^{\lambda_{i}}, i=$ $1, \ldots, n$, and let $Y_{1}, \ldots, Y_{n}$ be a random sample from a distribution with common survival distribution $\bar{F}^{\lambda}$. Khaledi and Kochar (2002) have shown that if $F$ is DFR (decreasing failure rate) then

$$
\lambda=\left(\prod_{i=1}^{n} \lambda_{i}\right)^{1 / n} \Longrightarrow Y_{n: n} \leq_{\operatorname{disp}} X_{n: n},
$$

which could actually be written as

$$
\lambda \geq\left(\prod_{i=1}^{n} \lambda_{i}\right)^{1 / n} \Longrightarrow Y_{n: n} \leq_{\operatorname{disp}} X_{n: n} .
$$

Motivated by this observation, we will extend Proposition 4.2 to the PHR model.

The following lemma due to Kochar et al. (2002) will be used to prove the result.

Lemma 4.1. Let $X$ and $Y$ be continuous random variables with finite means. Then, for any increasing and convex function $\phi$, it holds that

$$
Y \leq_{\mathrm{RS}} X \quad \Longrightarrow \phi(Y) \leq_{\mathrm{RS}} \phi(X)
$$

Theorem 4.1. Let $X_{1}, \ldots, X_{n}$ be independent random variables with $X_{i}$ having survival function $\bar{F}^{\lambda_{i}}, i=1, \ldots, n$, and let $Y_{1}, \ldots, Y_{n}$ be another random sample with common survival function $\bar{F}^{\lambda}$. If $F$ is DFR then

$$
\lambda^{*} \leq \lambda \quad \Longrightarrow \quad Y_{n: n} \leq \mathrm{RS} X_{n: n},
$$

where $\lambda^{*}$ is given in (4.3).

Proof. The proof is similar to Theorem 2.3 of Kochar and Xu (2007b). Note that the cumulative hazard of $F$ is

$$
H(x)=-\log \bar{F}(x)
$$

Now, for $x \geq 0$ and $i=1, \ldots, n$,

$$
\mathrm{P}\left(H\left(X_{i}\right)>x\right)=\mathrm{P}\left(X_{i}>H^{-1}(x)\right)=\bar{F}^{\lambda_{i}}\left(\bar{F}^{-1}\left(\mathrm{e}^{-x}\right)\right)=\mathrm{e}^{-\lambda_{i} x},
$$

where $H^{-1}$ is the right inverse of $H$. Letting $X_{i}^{\prime}=H\left(X_{i}\right)$, we note that $X_{i}^{\prime}$ is exponential with hazard rate $\lambda_{i}$ for $i=1, \ldots, n$. Similarly, let $Y_{i}^{\prime}=H\left(Y_{i}\right)$ be exponential with hazard rate $\lambda$ for $i=1, \ldots, n$. It follows from (4.3) that, for $\lambda^{*} \leq \lambda$,

$$
Y_{n: n}^{\prime} \leq_{\mathrm{RS}} X_{n: n}^{\prime}
$$

That is,

$$
H\left(Y_{n: n}\right) \leq \mathrm{RS} H\left(X_{n: n}\right) .
$$

Since $F$ is DFR, $H(\cdot)$ is increasing and concave, which implies that $H^{-1}(\cdot)$ is increasing and convex. The required result follows from Lemma 4.1.

As a consequence, we have the following result. 
Corollary 4.1. Let $X_{1}, \ldots, X_{n}$ be independent random variables with $X_{i}$ having survival function $\bar{F}^{\lambda_{i}}, i=1, \ldots, n$, and let $Y_{1}, \ldots, Y_{n}$ be another random sample with common survival function $\bar{F}^{\lambda}$. If $F$ is DFR then

$$
\lambda^{*} \leq \lambda \quad \Longrightarrow \quad \operatorname{var}\left(Y_{n: n}\right) \leq \operatorname{var}\left(X_{n: n}\right),
$$

where $\lambda^{*}$ is given in (4.3).

Remark. As a special case of $\bar{F}(x)=\mathrm{e}^{-x}$, Khaledi and Kochar (2000) gave a lower bound for the variance of the $X_{n: n}$ in terms of the geometric mean, i.e.

$$
\operatorname{var}\left(X_{n: n}\right) \geq \frac{1}{\hat{\lambda}} \sum_{i=1}^{n} \frac{1}{i^{2}} .
$$

Since the right-spread order implies that the variances are ordered (cf. Shaked and Shanthikumar (2007, p. 166)), Corollary 4.1 provides a better lower bound for the variance:

$$
\operatorname{var}\left(X_{n: n}\right) \geq \frac{1}{\lambda^{*}} \sum_{i=1}^{n} \frac{1}{i^{2}},
$$

as $\lambda^{*} \leq \lambda$.

\section{Acknowledgement}

The authors are grateful to an anonymous referee for constructive comments which led to an improved version of the paper.

\section{References}

Ahmed, A. N., Alzaid, A., Bartoszewicz, J. And Kochar, S. C. (1986). Dispersive and superadditive ordering. Adv. Appl. Prob. 18, 1019-1022.

Arnold, B. C. And Groeneveld, R. A. (1995). Measuring skewness with respect to the mode. Amer. Statistician 49, 34-38.

Barlow, R. E. AND Proschan, F. (1981). Statistical Theory of Reliability and Life Testing. Holt, Rinehart and Winston, New York.

Dykstra, R., Kochar, S. And Rojo, J. (1997). Stochastic comparisons of parallel systems of heterogeneous exponential components. J. Statist. Planning Infer. 65, 203-211.

Fernández-Ponce, J. M., Kochar, S. C. And Muñoz-Perez, J. (1998). Partial orderings of distributions based on right-spread functions. J. Appl. Prob. 35, 221-228.

Khaledi, B.-E. and Kochar, S. (2000). Some new results on stochastic comparisons of parallel systems. J. Appl. Prob. 37, 1123-1128.

Khaledi, B.-E. AND Kochar, S. (2002). Stochastic orderings among order statistics and sample spacings. In Uncertainty and Optimality, ed. J. C. Misra, World Scientific River Edge, NJ, pp. 167-203.

Kochar, S. C. (1989). On extensions of DMRL and related partial orderings of life distributions. Commun. Statist. Stoch. Models 5, 235-245.

Kochar, S. C. AND Wiens, D. P. (1987). Partial orderings of life distributions with respect to their aging properties. Naval Res. Logistics 34, 823-829.

Kochar, S. AND XU, M. (2007a). Some recent results on stochastic comparisons and dependence among order statistics in the case of PHR model. J. Iranian Statist. Soc. 6, 125-140.

Kochar, S. AND XU, M. (2007b). Stochastic comparisons of parallel systems when components have proportional hazard rates. Prob. Eng. Inf. Sci. 21, 597-609.

Kochar, S. C., LI, X. And ShaKed, M. (2002). The total time on test transform and the excess wealth stochastic order of distributions. Adv. Appl. Prob. 34, 826-845.

Marshall, A. W. And Olkin, I. (1979). Inequalities: Theory of Majorization and Its Application (Math. Sci. Eng. 143). Academic Press, New York. 
Marshall, A. W. And Olkin, I. (2007). Life Distributions. Springer, New York.

Mitrinović, D. S. (1970). Analytic Inequalities. Springer, Berlin.

Shaked, M. And Shantikumar, J. G. (1998). Two variability orders. Prob. Eng. Inf. Sci. 12, 1-23.

Shaked, M. And Shanthikumar, J. G. (2007). Stochastic Orders. Springer, New York.

VAN Zwet, W. R. (1970). Convex Transformations of Random Variables (Math. Centre Tracts 7), 2nd edn. Mathematical Centre, Amsterdam. 